\title{
ON NEAR-RINGS OF QUOTIENTS
}

\author{
by A. OSWALD \\ (Received 1st April 1977)
}

\section{Introduction}

In (2), Holcombe investigated near-rings of zero-preserving mappings of a group $\Gamma$ which commute with the elements of a semigroup $S$ of endomorphisms of $\Gamma$ and examined the question: under what conditions do near-rings of this type have near-rings of right quotients which are 2-primitive with minimum condition on right ideals? In the first part of this paper (\$2) we investigate further properties of near-rings of this type. The second part of the paper (\$3) deals with those near-rings which have semisimple near-rings of right quotients. Our results here are analogous to those of Goldie (1); in particular, with a suitable definition of finite rank we prove that a near-ring which has a semisimple near-ring of right quotients has finite rank.

Basic concepts for near-rings can be found in Pilz (5). All our near-rings will be left near-rings (i.e. $a(b+c)=a b+a c$ for all $a, b, c \in N$ ) with a two-sided zero.

\section{Near-rings of quotients of mapping near-rings}

If $S$ is a semigroup, a set $A$ is an $S$-set if it admits the elements of $S$ as right operators with $a\left(s_{1} s_{2}\right)=\left(a s_{1}\right) s_{2}$ for all $a \in A, s_{1}, s_{2} \in S$. A mapping $f: A \rightarrow B$, where $A$ and $B$ are $S$-sets, is an $S$-homomorphism if for each $a \in A$ and $s \in S$ we have (af) $s=($ as ) $f$. If $X \subset A$, an $S$-set, and every mapping $f: X \rightarrow B$, where $B$ is an $S$-set, defines a unique $S$-homomorphism $f^{*}: A \rightarrow B$ then $A$ is the free $S$-set on $X$.

If $S$ has a semigroup $G$ of two-sided quotients we define $A G=\{a g: a \in A, g \in G\}$. Denoting by $C$ the set of cancellative elements of $S$ it is obvious that $A G=$ $\left\{a s^{-1}: a \in A, s \in C\right\}$. In $A G$ we define $a s^{-1}=b t^{-1}$ if, and only if, for some $u, v \in C$ we have $s u=t v$ and $a u=b v$. Then in $A G$ we can define an action by $(a g) h=a(g h)$ whenever $a \in A, g, h \in G$ and $A G$ becomes a $G$-set.

The following result is well known.

Lemma 0. If a semigroup $X$ has a semigroup of right quotients and if $C$ denotes the subsemigroup of $X$ consisting of the cancellative elements then for each set $t_{1}, t_{2} \ldots, t_{k} \in C$ there exist $s_{1}, s_{2}, \ldots, s_{k}, t \in C$ with $t=s_{i} t_{i}, 1 \leqslant i \leqslant k$.

Proof. See (6; Lemma 1.4), for example.

A similar result holds if $X$ has a semigroup of left quotients.

Now suppose that $A$ is a group (written additively but not necessarily com- 
mutative) and that $S$ is a semigroup of endomorphisms of $A$ in which the cancellative elements of $S$ are monomorphisms of $A$. We suppose the zero endomorphism is not in $S$. If $S$ has a semigroup $G$ of two-sided quotients we can define an addition in $A G$ by $\left(a s^{-1}+b t^{-1}\right)=(a u+b v) w^{-1}$ where $u, v \in C$ with $w=s u=t v$. It is easy to show that $A G$ is a group and that the elements of $G$ act as endomorphisms of $A G$.

Now we introduce the two sets

$$
\begin{gathered}
M=\operatorname{Map}_{s}(A)=\{f: A \rightarrow A: 0 f=0,(a f) s=(a s) f, a \in A, s \in S\} \\
M^{*}=\operatorname{Map}_{G}(A G)=\left\{f: A G \rightarrow A G: 0 f=0,\left(\left(a g_{1}\right) f\right) g_{2}=\left(a g_{1} g_{2}\right) f, a g_{1} \in A G, g_{2} \in G\right\} .
\end{gathered}
$$

It is easy to see that both $M$ and $M^{*}$ are left near-rings. Furthermore, if $f \in M$ we can define $f^{*} \in M^{*}$ by $\left(a s^{-1}\right) f^{*}=(a f) s^{-1}$. Since it is easily seen that the mapping $f \rightarrow f^{*}$ is an injective near-ring homomorphism we see that $M$ is embedded in $M^{*}$. We identify $f$ with $f^{*}$ in $M^{*}$. We wish to introduce restrictions on $A, S$ and $A G$ which will lead to $M^{*}$ being a near-ring of quotients of $M$.

We say that $A$ is a torsion-free $S$-set if whenever $a \in A$ with $a \neq 0$ and $s, t \in S$ with $s \neq t$ then $a s \neq a t$. Using Lemma 0 it can be shown that if $A$ is a torsion-free $S$-set then $A G$ is a torsion-free $G$-set. Furthermore, we call $A$ finitely-generated if for some set $a_{1}, a_{2}, \ldots, a_{n} \in A$ we have $A=a_{1} S \cup a_{2} S \cup \ldots \cup a_{n} S$. Then clearly $A G=$ $a_{1} G \cup a_{2} G \cup \ldots \cup a_{n} G$. Restricting ourselves to the case when $A G$ is a free $G$-set we can choose $a_{i_{1}}, a_{i_{2}}, \ldots, a_{i_{k}}$ with $A G=a_{i_{1}} G \dot{U} a_{i_{2}} G \dot{U} \ldots \dot{U} a_{i_{k}} G$ where distinct terms in this union are disjoint. Since $A \subset A G, a_{j}=a_{\lambda_{j}} r_{j}^{-1} s_{j}$ for some $\lambda_{j} \in\left\{i_{1}, \ldots, i_{k}\right\}$ and $r_{j} \in C$, $s_{j} \in S$. Using Lemma 0 choose $e_{j}, d \in C$ with $r_{j}^{-1}=d^{-1} e_{j}$. Then $a_{j} \in a_{\lambda_{j}} d^{-1} S$ and so $A \subseteq a_{i_{1}} d^{-1} S \cup a_{i_{2}} d^{-1} S \dot{\cup} \ldots \dot{\cup} a_{i_{k}} d^{-1} S$ as a disjoint union and also $A G=$ $a_{i_{1}} d^{-1} G \dot{U} \ldots \dot{\cup} a_{i_{k}} d^{-1} G$. For $h \in G$ we define $\mu_{h}: A G \rightarrow A G$ by $\left(a_{i_{j}} d^{-1} q\right) \mu_{h}=a_{i_{i}} d^{-1} h q$ where $q \in G$ and $1 \leqslant j \leqslant k$. Then $\mu_{h} \in M^{*}$ and if $h \in C$ then $\mu_{h}$ has an inverse in $M^{*}$ and $\left(\mu_{h}\right)^{-1}=\mu_{h}^{-1}$.

Proposition 1. The elements of $M^{*}$ have the form $\theta \mu_{t}^{-1}$ for some $\theta, \mu_{t} \in M$. Let

Proof. If $f \in M^{*}$ then $f$ is uniquely determined by its effect upon $a_{i j} d^{-1}, 1 \leqslant j \leqslant k$.

$$
a_{i_{j}} d^{-1} f=a_{\lambda_{j}} d^{-1} p_{j}^{-1} s_{j}, p_{j} \in C, s_{j} \in S, i_{i}, \lambda_{j} \in\left\{i_{1}, i_{2}, \ldots, i_{k}\right\}
$$

Applying the symmetric form of Lemma 0 we can choose $q_{1}, \ldots, q_{k}, r \in C$ with $p_{j}^{-1}=r^{-1} q_{j}, 1 \leqslant j \leqslant k$ and then

$$
a_{i j} d^{-1} f=a_{\lambda_{j}} d^{-1} r^{-1} q_{j} s_{j} \text {. }
$$

Then $a_{i_{j}} d^{-1} f \mu_{d r}=a_{\lambda_{j}} q_{j} s_{j} \in A$ so that $f \mu_{d r}: a_{i_{1}} d^{-1} S \dot{U} \ldots \dot{U} a_{i_{k}} d^{-1} S \rightarrow A$ and so $f \mu_{d r}: A \rightarrow$ $A$ and $f \mu_{d r} \in M$. Also $a_{i j} d^{-1} \mu_{d r}=a_{i_{j}} r \in A$ so $\mu_{d r} \in M$ and writing $\theta=f \mu_{d r}$ and $t=d r \in$ $C$ we have $f=\theta \mu_{t}^{-1}$ as required.

It is easy to see that if $t \in C$ and $\mu_{t} \in M$ then $\mu_{t}$ is cancellative in $M$.

Proposition 2. If $f \in M$ is cancellative then $f$ is cancellative in $M^{*}$.

Proof. Let $\phi, \psi \in M^{*}$ and $f \phi=f \psi$. Write $a_{j}^{*}=a_{i_{j}} d^{-1}$. If $\phi \neq \psi$ let $a_{j}^{*} \phi \neq a * \psi$. If 
$a \in A$ and $s \in S$ with $a f=a_{j}^{*} s$ then $a_{j}^{*} \psi s=a_{j}^{*} s \psi=a f \psi=a f \phi=a_{j}^{*} \phi s \quad$ and since $A G$ is torsion-free and $s \in G$ we see that $a_{j}^{*} \psi=a_{i}^{*} \phi$ which is false. Consequently there is no $a \in A$ with $a f=a_{j}^{*} s$ for any $s \in S$. Let $\lambda_{j}: A G \rightarrow A G$ be defined by

$$
a_{i}^{*} u v^{-1} \lambda_{j}= \begin{cases}0 & \text { if } i \neq j \\ a_{j}^{*} u v^{-1} & \text { if } i=j\end{cases}
$$

where $u v^{-1} \in G$. Then $\lambda_{j} \in M^{*}$ for $1 \leqslant j \leqslant k$. Then for each $a \in A$ af $\lambda_{j}=0=a f 0$ so that in $M$ we have $f\left(\left.\lambda_{j}\right|_{A}\right)=f 0$ and $\left.\lambda_{j}\right|_{A}=0$. But then $a_{j}^{*} \lambda_{j}=\left(\left.a_{i} \lambda_{j}\right|_{A}\right) d^{-1}=0$ and so $f \phi=f \psi$ implies $\phi=\psi$. Now suppose $\phi f=\psi f$ and $\phi \neq \psi$. Without loss of generality we can take $a_{1}^{*} \phi \neq a_{1}^{*} \psi$. Set $a_{1}^{*} \phi=a_{1}^{*} \alpha \beta^{-1}, a_{1}^{*} \psi=a_{j}^{*} \gamma \delta^{-1}$ where $\alpha \beta^{-1}, \gamma \delta^{-1} \in G$. Then

$$
a_{i}^{*} f \alpha \beta^{-1}=a_{i}^{*} \alpha \beta^{-1} f=a_{i}^{*} \phi f=a_{1}^{*} \psi f=a_{i}^{*} \gamma \delta^{-1} f=a_{i}^{*} f \gamma \delta^{-1} .
$$

If $i=j$ either $\alpha \beta^{-1}=\gamma \delta^{-1}$ or $a_{i}^{*} f=0$ since $A G$ is torsion-free. If $\alpha \beta^{-1}=\gamma \delta^{-1}$ then $a_{1}^{*} \phi=a_{i}^{*} \alpha \beta^{-1}=a_{i}^{*} \gamma \delta^{-1}=a_{1}^{*} \psi$ which is false. Hence $a_{i}^{*} f=0$. In this case $a \lambda_{i} f=0$ for each $a \in A$ so that in $M$ we have $\left(\left.\lambda_{i}\right|_{A}\right) f=0 f$ and $\left.\lambda_{i}\right|_{A}=0$ which is false. Thus $i \neq j$. In this case let $a_{i}^{*} f=\left(a_{p}^{*} t^{-1}\right) s, a_{j}^{*} f=\left(a_{q}^{*} t^{-1}\right) s_{1}$ where $s, s_{1} \in S$ and we have used Lemma 0 to find $t \in C$. Then

$$
\begin{aligned}
& a_{p}^{*} t^{-1} s \alpha \beta^{-1}=a_{i}^{*} f \alpha \beta^{-1}=a_{i}^{*} \alpha \beta^{-1} f=a_{1}^{*} \phi f \\
& a_{q}^{*} t^{-1} s_{1} \gamma \delta^{-1}=a_{j}^{*} f \gamma \delta^{-1}=a_{j}^{*} \gamma \delta^{-1} f=a_{j}^{*} \psi f
\end{aligned}
$$

and so $a_{p}^{*} t^{-1} s \alpha \beta^{-1}=a_{q}^{*} t^{-1} s_{1} \gamma \delta^{-1} \in a_{p}^{*} G \cap a_{q}^{*} G$ and thus $p=q$. Hence $a * f, a * f \in a_{p}^{*} G$ and so for some $r, 1 \leqslant r \leqslant k, a_{r}^{*} G \cap \operatorname{Im} f=\phi$. Again we have the situation where for no $a \in A$ is $a f=a_{r}^{*} s$ for any $s \in S$ and again we deduce that $\lambda_{r}=0$. Hence $\phi=\psi$ is cancellative in $M^{*}$.

Proposition 3. If $f \in M^{*}$ and $f$ is cancellative then $f$ has an inverse in $M^{*}$.

Proof. If $f$ is both $1-1$ and onto then the inverse mapping $f^{-1}: A G \rightarrow A G$ exists and a simple calculation shows that $f^{-1} \in M^{*}$. Suppose $f$ is cancellative but not onto. Let $b \in A G$ with $b \notin \operatorname{Im} f$. For some $j, b=a_{j}^{*} g$ with $g \in G$ and so $a_{l}^{*} \notin \operatorname{Im} f$. But then, for $1 \leqslant i \leqslant k, a_{i}^{*} f \lambda_{j}=0$ and so $f \lambda_{j}=0$ and $\lambda_{j}=0$ since $f$ is cancellative. Hence $f$ is onto. Now suppose $a, b \in A G$ with $a f=b f, a \neq b$. For some $i, j, a=a_{i}^{*} g_{1}, b=a_{j}^{*} g_{2}$ with $g_{1}, g_{2} \in G$. If $i \neq j$ then $a_{i}^{*} g_{1} f=a_{j}^{*} g_{2} f$ and so $a_{i}^{*} f g_{1}=a_{i}^{*} f g_{2}$. Also for some $h, r$ we have $a_{*}^{*} f \in a_{h}^{*} G, a_{j}^{*} f \in a_{r}^{*} G$ and $a_{i}^{*} f g_{1} \in a_{h}^{*} G \cap a_{r}^{*} G \neq \phi$. Since $A G$ is free we have $h=r$ and thus both $a_{i}^{*}$ and $a_{j}^{*}$ map into $a_{h}^{*} G$. But then $f$ cannot be onto. This contradiction establishes the result.

These results now lead to

Theorem 1. Let $S$ be a semigroup with a semigroup $G$ of two-sided quotients and let $A$ be a group for which $S$ is a semigroup of endomorphisms such that the cancellative elements of $S$ are monomorphisms. If $A$ is torsion-free and finitely generated as an $S$-set and if $A G$ is free as a $G$-set then $M$ has a near-ring of two-sided quotients which is isomorphic to $M^{*}$. 
Proof. It remains only to prove that $M^{*}$ is a near-ring of left quotients of $M$. Let $f \in M^{*}$. The for some $\alpha_{i}, \bar{\alpha}_{i} \in S, \beta_{i}, d_{i} \in C a_{\lambda_{i}} d^{-1} f=a_{\lambda_{j(i)}} d^{-1} \alpha_{i} \beta_{i}^{-1}=a_{\lambda_{j i}} \bar{\alpha}_{i}\left(\beta_{i} d_{i}\right)^{-1}$ since $G$ is a semigroup of two-sided quotients of $S$. Using Lemma 0 we have, for $1 \leqslant i \leqslant k$, $\left(\beta_{i} d_{i}\right)^{-1}=e_{i} r^{-1}$ and $d^{-1}=e r^{-1}$ for some $e_{i}, e, r \in C$. Then $a_{\lambda_{i}} d^{-1} f r=a_{\lambda_{i(i)}} \bar{\alpha}_{i} e_{i} \in A$. But $a_{\lambda_{i}} d^{-1} f r=a_{\lambda_{i}} d^{-1} r f=a_{\lambda_{i}} d^{-1} \mu_{r} f$ and so $\mu_{r} f \in M$. Also $a_{\lambda_{i}} d^{-1} \mu_{r}=a_{\lambda_{i}} d^{-1} r=a_{\lambda_{i}} e \in A$ and so $\mu_{r} \in M$. Since $r \in C$ we have $f=\left(\mu_{r}\right)^{-1} \theta$ for some $\theta \in M$ as required.

We now turn to the structure of $M^{*}$. Since $A G$ is free, $A G=$ $a_{\lambda_{1}} d^{-1} G \dot{U} \ldots \dot{\cup} a_{\lambda_{k}} d^{-1} G$. Writing $a_{j}^{*}=a_{\lambda_{j}} d^{-1}$ we have defined $\lambda_{i}: A G \rightarrow A G$ by $a_{i}^{*} g \lambda_{i}=$ 0 if $j \neq i$ and $a_{i}^{*} g \lambda_{i}=a_{i}^{*} g$ where $g \in G$. Clearly $\lambda_{i} \in M^{*}$ and it is an easy calculation to show that, for each $i, \lambda_{i} M^{*}$ is a right ideal of $M^{*}$ and that $M^{*}=\lambda_{1} M^{*} \oplus \ldots \oplus \lambda_{k} M^{*}$.

For the remainder of this section we will suppose that $G$ is a group. If $X \subset N$, where $N$ is a near-ring, we denote by $r(X)$ the set $r(X)=\{n \in N: x n=0$ for all $x \in X\}$; by $r(x)$ we mean $r(\{x\})$.

Theorem 2. $M^{*}$ has no proper two-sided ideals and has the descending chain condition on $M^{*}$-subgroups.

Proof. If $\lambda_{i} g, \lambda_{i} h \in \lambda_{i} M^{*}$ with $\lambda_{i} g \neq 0 \neq \lambda_{i} h$ then $a_{i}^{*} \lambda_{i} h=a_{m}^{*} \alpha \beta^{-1}$ for some $m$ and $\alpha \beta^{-1} \in G$ and $a_{j}^{*} \lambda_{i} h=0$ for $j \neq i$. Similarly for some $p$ and $\gamma \delta^{-1} \in G, a_{i}^{*} \lambda_{i} g=a_{p}^{*} \gamma \delta^{-1}$, $a_{j}^{*} \lambda_{i} g=0$ if $j \neq i$. Defining $q: A G \rightarrow A G$ by $a_{p}^{*} q=a_{m}^{*} \alpha \beta^{-1} \gamma \delta^{-1}$ and $a_{i}^{*} q=0$ if $j \neq p$ we can extend $q$ to $q^{*} \in M^{*}$ to get $\lambda_{i} h=\lambda_{i} g q^{*} \in \lambda_{i} g M^{*}$ and so $\lambda_{i} h M^{*}=\lambda_{i} g M^{*}$. Hence each $\lambda_{i} M^{*}$ is minimal. Thus $M^{*}$ is completely reducible with identity and by (3) $M^{*}$ has no nilpotent $M^{*}$-subgroups and the descending chain condition on $M^{*}$ subgroups. Next we let $f \in r\left(\lambda_{1} M^{*}\right)$. Defining $\lambda_{1 j} \in M^{*}$ by $a_{i}^{*} \lambda_{1 j}=0$ if $i \neq 1$ and $a_{1}^{*} \lambda_{1 j}=a_{j}^{*}$ we see that $f=0$. From (4; Thm 3) we can write $M^{*}$ as a direct sum of two-sided ideals each of which is simple as a near-ring. If $U, V$ are two such with $U \cap V=(0)$ then $U V=0$. If $U \neq 0, \lambda_{1} M^{*} U \neq 0$ so for some $f \in M^{*}, \lambda_{1} f U=\lambda_{1} M^{*}$. But then $\lambda_{1} M^{*} V=0$ so $V=0$. It follows that $M^{*}$ is simple as a near-ring.

Suppose $X$ is a subset of the near-ring $N$. Set $X_{0}=N X \cup X$ and denote by $X_{0}^{+}$the normal subgroup of $N$ generated by $X_{0}$. Let $\left[X_{0}^{+}\right]=\left\{(a+x) c-a c: x \in X_{0}^{+}, a, c \in N\right\}$. Define $X_{1}=\left[X_{0}^{+}\right] \cup X_{0}^{+}$and observe that $N X_{1} \subseteq X_{1}$, We now suppose that $X_{k}$ has been constructed and we construct successively $X_{k}^{+},\left[X_{k}^{+}\right]$and $X_{k+1}=X_{k}^{+} \cup\left[X_{k}^{+}\right]$. This leads to a chain $X_{0} \subseteq X_{1} \subseteq \ldots$ and we then define $I(X)=\cup X_{k}$. A simple calculation shows that $I(X)$ is the ideal generated by $X$.

If $N$ has a near-ring $Q$ of two-sided quotients and $B$ is an $N$-subgroup of $N$ then we may construct the ideal $B^{*}$ of $Q$ generated by $Q B Q$. In this case $B^{*}=\cup B_{k}$ where $B_{0}=Q B Q$, etc.

Lemma 1. If $A$ is an $N$-subgroup with $A B=(0)$ and $A \neq(0)$ then for each $k$ the elements of $B_{k}$ have the form $c^{-1} t$ where $c$ is a cancellative element in $N$ and $A t=(0)$.

Proof. If $u \in Q B Q$ then $u=c^{-1} v b q$ where $v \in N, b \in B, q \in Q$ and $c$ is a cancellative element of $N$. Then $a v b q \in A B q=(0)$ and $u=c^{-1} t$ as required. Now suppose the result is true for $B_{k}$. If $u \in B_{k+1}$ then $u \in B_{k}^{+}$or $u \in\left[B_{k}^{+}\right]$. In the first case $u=\Sigma\left(d_{i}^{-1} x_{i}+c_{i}^{-1} t_{i}-d_{i}^{-1} x_{i}\right)=c^{-1} \Sigma\left(y_{i}+r_{i} t_{i}-y_{i}\right)$, on applying Lemma 0 , where $y_{i}, r_{i} \in N$ and $A t_{i}=(0)$. But then $A r_{i} t_{i}=(0)$ so with $t=\Sigma\left(y_{i}+r_{i} t_{i}-y_{i}\right)$ we have $u=c^{-1} t$ and $A t=(0)$. A similar argument applies to $\left[B_{k}^{+}\right]$and the lemma follows. 
Theorem 3. If $N$ has a near-ring $Q$ of two-sided quotients which is simple then $N$ is strictly prime.

Proof. Let $A, B$ be $N$-subgroups of $N$ with $A B=(0)$ but $A \neq(0)$. Let $B^{*}$ be the ideal of $Q$ generated by $Q B Q$. Since $Q$ is simple either $B^{*}=(0)$ or $B^{*}=Q$. If $B^{*}=(0)$ then trivally $B=(0)$. If $B^{*}=Q$ then $1 \in B^{*}$ so $1 \in B_{k}$ for some $k$. From Lemma 1 , $1=c^{-1} t$ with $c$ cancellative and $A t=(0)$. Thus $A c=(0)$ contrary to $A \neq(0)$.

Corollary 1. If $G$ is a group $M$ is strictly prime.

\section{Semisimple Near-rings of Right Quotients}

We now turn to an investigation of the properties of those near-rings which have near-rings of right quotients which are semisimple. If $Q$ is a near-ring then $Q$ is semisimple if it has no nilpotent $Q$-subgroups and the minimum condition on $Q$ subgroups. To fix our terminology, an $N$-subgroup $A$ is nilpotent if $A \neq(0)$ but for some $n \geqq 2, A^{n}=\left\{a_{1} a_{2} \ldots a_{n}: a_{i} \in A\right\}=(0)$.

Most of our results are the analogues of those of Goldie. We will determine necessary conditions for the near-ring $Q$ of right quotients of $N$ to be semisimple. It seems most unlikely, however, that these conditions will be sufficient. Throughout this section $Q$ will denote a near-ring of right quotients of $N$.

Theorem 4. If $N$ has a near-ring $Q$ of right quotients which is sermisimple then $N$ has no nilpotent $\mathbf{N}$-subgroups.

Proof. Let $A$ be a nilpotent $N$-subgroup of $A$ with $A^{n}=(0)$. Set $P=\ell(N A)=$ $\{x \in N: x N A=(0)\}$. If $u \in N(u \neq 0)$ with $u N \neq(0)$ and $P \cap u N=(0)$ then $N A \cap$ $u N \neq(0)$ so we can take $u x \in N A \cap u N$ with $u x \neq 0$. For some $k>1,(N A)^{k}=(0)$, $(N A)^{k-1} \neq(0)$. Hence $u x(N A)^{k-1}=(0)$. It follows that $(u x)^{k-1} N A=(0)$ and $(u x)^{k-1} \in$ $P \cap u N=(0)$. Thus $(u x)^{k-1}=0$. If $(u x)^{k-2} N A \neq(0)$ then for some $v \in N A,(u x)^{k-2} v \neq 0$ but $(u x)^{k-2} v N A=(0)$ so that $(u x)^{k-2} v \in P \cap u N=(0)$ which is a contradiction. Hence $P \cap u N \neq(0)$ and $P$ has non-empty intersection with every non-zero $N$-subgroup. Now consider $P Q$. Clearly if $q \in Q$ then $P Q \cap q Q \neq(0)$ unless $q=0$. In particular $P Q \cap e_{i} Q \neq(0)$ where $e_{i} Q$ is a minimal right ideal of $Q$ and $Q=$ $e_{1} Q \oplus e_{2} Q \oplus \ldots \oplus e_{n} Q$. Hence $e_{i} Q=p_{i} Q$ for some $p_{i} \in P$. Notice that $p_{1} N+p_{2} N+$ $\cdots+p_{n} N$ is an $N$-subgroup of $N$ and that $\left(p_{1} a_{1}+p_{2} a_{2}+\cdots+p_{n} a_{n}\right) b=$ $p_{1} a_{1} b+p_{2} a_{2} b+\cdots+p_{n} a_{n} b$ for $b \in N$. Thus $p_{1} N+p_{2} N+\cdots+p_{n} N \subseteq P$. However, $1=p_{1} a_{1} c_{1}^{-1}+\cdots+p_{n} a_{n} c_{n}^{-1}=\left(p_{1} a_{1} b_{1}+\cdots+p_{n} a_{n} b_{n}\right) c^{-1}$ where $a_{n}, b_{n} \in N$ and we have used Lemma 0 . It follows that $c \in P$ and $P$ contains a cancellative element. Hence $N A=(0)$ and $A=(0)$ as required.

A near-ring $N$ is strictly semiprime if it has no nilpotent $N$-subgroups. Thus

Corollary 2. Q semisimple implies $N$ strictly semiprime.

We say that an $N$-subgroup $A$ of $N$ is module-essential if, whenever $X$ is non-zero right ideal of $N$, then $A \cap X \neq(0) ; A$ is $N$-essential if whenever $X$ is a non-zero $N$-subgroup of $N$ then $A \cap X \neq(0)$. Certainly if $A$ is $N$-essential then $A$ is 
module essential. However, it is not generally true that if $A$ is module-essential then $A$ is $\boldsymbol{N}$-essential.

Theorem 5. If $Q$ is semisimple then module essential $N$-subgroups of $N$ are $N$-essential.

Proof. Let $X$ be module-essential and consider $X A$. If $e Q$ is a minimal right ideal of $Q$ then $X \cap e Q \cap N \neq(0)$ so $X Q \cap e Q \neq(0)$. As in the proof of Theorem 4 we now deduce that $X Q=Q$. If $a \in N(a \neq 0)$ then $a \in Q=X Q$ so $a=x c^{-1}$ where $x \in X$ and $c$ is cancellative. It follows that $a c \in X \cap a N \neq(0)$ and $X$ is $N$-essential.

We denote by $Z(N)$ the set $\{x \in N: r(x)$ is $N$-essential in $N\}$. The proof of Theorem 5 can be modified to show that each $N$-essential $N$-subgroup contains a cancellative element when $Q$ is semisimple and we deduce

Corollary 3. If $Q$ is semisimple then $Z(N)=(0)$.

In the usual way we prove

Theorem 6. If $Q$ is semisimple then $N$ has the maximum condition on right annihilators.

We say that a near-ring $N$ has finite rank if each chain $A_{1} \subset A_{2} \subset \ldots$ of $N$ subgroups in which for each $i \geqq 2$ there is a non-zero $N$-subgroup $B_{i} \subset A_{i}$ with $B_{i} \cap A_{i-1}=(0)$ terminates finitely. If $N$ is a ring this reduces to the usual definition.

Lemma 2. If $Q$ is semisimple and $A_{1} \subset A_{2} \subset \ldots$ is a chain of $N$-subgroups of $N$ such that for each $i \geqslant 2$ there is a non-zero $N$-subgroup $B_{i} \subset A_{i}$ with $B_{i} \cap A_{i-1}=(0)$ then $B_{i}$ can be chosen to be a submodule of the $N$-module $A_{i}$.

Proof. Let $X$ be a right ideal of $N$ maximal subject to the condition $A_{i-1} \cap X=$ (0). Then $A_{i-1}+X$ is module-essential in $N$ so by Theorem $5, A_{i-1}+X$ is $N$-essential. Now $A_{i} \cap\left(A_{i-1}+X\right)=A_{i-1}+(A \cap X)$ and $B_{i} \cap A_{i} \cap\left(A_{i-1}+X\right) \neq(0)$ so that $A_{i} \cap$ $X \neq(0)$. Clearly $A_{i} \cap X$ is a submodule of $A_{i}$ with $A_{i} \cap X \cap A_{i-1}=(0)$.

We wish to prove that a near-ring with a semisimple near-ring of right quotients has finite rank. Because the form of a $Q$-subgroup generated by an $N$-subgroup of $N$ does not have a simple representation in the form $I Q$, say, we have to adopt a more indirect approach. We begin by showing that there are essentially two different problems to be solved.

From (4; Thm 3) a semisimple near-ring $Q$ with identity is the direct sum of finitely many two-sided ideals each of which is simple as a near-ring. We divide these direct summands into two classes, those which are rings and those which are non-rings i.e. near-rings which are not rings. Write $Q=R \oplus T$ where $R, T$ are ideals of $Q, R$ is the sum of those direct summands which are rings and $T$ the sum of those which are non-rings. Clearly $R$ is a ring. Define $N_{1}=N \cap R, N_{2}=N \cap T$. Then $N_{1}, N_{2}$ are ideals of $N$ and $N_{1}$ is a ring. Clearly 
Lemma 3. As a right ideal $N_{1} \oplus N_{2}$ is $N$-essential in $N$.

Lemma 4. As near-rings $R$ and $T$ are each semisimple with an identity.

We now prove the following result.

Theorem 7. Let $Q$ be a semisimple near-ring of right quotients of the near-ring $N$ and suppose that $I$ is an ideal of $Q$. Then $I$ is a semisimple near-ring of right quotients of the near-ring $J=I \cap N$.

Proof. From (4; Corollary 6) there is an ideal $X$ of $Q$ with $I \cap X=(0), I \oplus X=$ Q. As before $J \oplus X \cap N$ is essential as a right ideal in $N$. Clearly $J \subseteq I$ and $I$ has an identity. Write $Y=X \cap N$. Let $d \in J$ be cancellative in $J$ and $g \in Y$ be cancellative in $Y$. Suppose $u \in N$ with $(d+g) u=0$. There is a cancellative $c \in N$ with $u c \in J \oplus Y$ (unless $u=0$ ). Also $u c=n_{1}+n_{2} ; n_{1} \in J, n_{2} \in Y$ and $0=(d+g)\left(n_{1}+n_{2}\right)=d n_{1}+g n_{2}$ so $d n_{1}=0=g n_{2}$ and $n_{1}=n_{2}=0$ from which we deduce $u c=0$ and $u=0$. It follows that $d+g$ is right cancellative in $N$ and $r_{Q}(d+g)=0$. Write $p=d+g$. Then $p Q \supseteq p^{2} Q \supseteq$ $\ldots$ and thus $p^{n} Q=p^{n+1} Q$ for some $n$. But then $p^{n}=p^{n+1} q$ for some $q \in Q$ and $p^{n-1}-p^{n} q \in r_{Q}(p)=(0)$. Continuing in this way we get $1=p q$ for some $q \in Q$. If $u_{1}$, $u_{2} \in N$ with $u_{1}(d+g)=u_{2}(d+g)$ then, in $Q, u_{1} p=u_{2} p$ and $u_{1} p q=u_{1}=u_{2} p q=u_{2}$. We see that $d+g$ is cancellative in $N$. In $Q,(d+g)^{-1}=\alpha+\beta$ where $\alpha \in I, \beta \in X$ and $1=(d+g)(\alpha+\beta)=d \alpha+g \beta$. Thus $d \alpha$ is the identity of $I$ and $d^{-1}=\alpha \in I$. Now let $r \in I$. For some $a, b \in N$ with $b$ cancellative $r=a b^{-1}$ and $r b=a \in I \cap N=J$. For some cancellative $c_{1} \in N, b c_{1} \in J \oplus Y$ so $b c_{1}=u_{1}+v_{1}, u_{1} \in J, v_{1} \in Y$. Since $b c_{1}$ is cancellative in $N, u_{1}$ is cancellative in $J$ and $c_{1}^{-1} b^{-1}=u_{1}^{-1}+v_{1}^{-1}, u_{1}^{-1} \in I, v_{1}^{-1} \in X$. But then $a c_{1} c_{1}^{-1} b^{-1}=a c_{1} u_{1}^{-1}+a c_{1} v_{1}^{-1}$ and $a c_{1} \in I$ so $a c_{1} v_{1}^{-1}=(0)$. Thus $r=a c_{1} u_{1}^{-1}$ where $a c_{1}, u_{1} \in J$ and $u_{1}$ is cancellative in $J$. Hence $I$ is a near-ring of right quotients of $J$ and clearly $I$ is semisimple with identity.

Corollary 4. $N_{1}$ has $R$ as a semisimple ring of right quotients and $N_{2}$ has $T$ as semisimple near-ring of right quotients.

Now let $A_{1} \subset A_{2} \subset \ldots$ be a chain of $N$-subgroups in which, for each $i \geqq 2$, there is a non-zero $N$-subgroup $B_{i} \subset A_{i}$ with $A_{i-1} \cap B_{i}=(0)$. Since $N_{1} \oplus N_{2}$ is $N$-essential in $N$ as a right ideal we can suppose that $A_{i} \subset N_{1} \oplus N_{2}$ for each $i$ and for convenience we take $N=N_{1} \oplus N_{2}$. There are then the following possibilities:-

(i) infinitely many $A_{i} \subset N_{2}$;

(ii) only finitely many $A_{i} \subset N_{2}$;

(a) for infinitely many $i, B_{i}$ can be chosen with $B_{i} \cap N_{2} \neq(0)$,

(b) for only finitely many $i$ can we choose $B_{i}$ with $B_{i} \cap N_{2} \neq(0)$.

In the case (ii) (a) we can consider the chain $A_{1} \cap N_{2} \subset A_{2} \cap N_{2} \subset \ldots$ and we are back with case (i).

Lemma 5. If case (ii) (b) arises then the chain $A_{1} \subset A_{2} \subset \ldots \subset A_{n} \subset \ldots$ is finite.

Proof. For convenience we suppose that for each possible choice of $B_{i}$ we have 
$B_{i} \cap N_{2}=(0)$. Then $A_{i} \cap N_{2}$ is $N$-essential in $A_{i+1} \cap N_{2}$ for each $i$. Pass to $\bar{N}=N / N_{2} \cong$ $N_{1}$. Then $\bar{A}_{i} \subset \bar{A}_{i+1}, \bar{B}_{i+1} \subset \bar{A}_{i+1}$ and if $\bar{u} \in \bar{A}_{i} \cap \bar{B}_{i+1}$ then $u \in\left(A_{i}+N_{2}\right) \cap\left(B_{i+1}+N_{2}\right)$ and so $u=a+n=b+n^{\prime}$ where $a \in A_{i}, b \in B_{i+1}, n, n^{\prime} \in N_{2}$. Now $-b+a=n^{\prime}-n \in$ $N_{2} \cap A_{i+1}$ so for some cancellative $c \in N,(-b+a) c \in A_{i} \cap N_{2}$. In view of Lemma 2, we can choose $B_{i+1}$ to be a submodule of $A_{i+1}$ and then $(-b+a) c-a c \in$ $B_{i+1} \cap A_{i} \cap N_{2}=(0)$. Hence $b=0$ and $\bar{u}=\overline{0}$. But then $\bar{B}_{1} \oplus \bar{B}_{2} \oplus \ldots$ is a direct sum of right ideals of a ring with a semisimple ring of right quotients so by Goldie's Theorem the sum is finite. It follows that the chain $A_{1} \subset A_{2} \subset \ldots$ terminates finitely.

For case (i) we will need the following results.

Theorem 8. If $R$ is a semisimple near-ring with identity and $A$ is a maximal right annihilator then $A=r(e)$ for some idempotent $e$ with $e R$ a minimal $R$-subgroup of $R$.

Proof. Since $A \neq R$ we let $U$ be a right ideal of $R$ with $A \cap U=(0), A+U=R$. If $\ell(A) \cap U=(0)$ then $U \ell(A) \subseteq U \cap \ell(A)=(0)$ so if $m \in \ell(A), U m=(0)$ and hence $(m U)^{2}=(0)$. It follows that $m U=(0)$ and $U \subseteq r \ell(A)=A$. Since $A \cap U=(0)$ this contradiction establishes that $\ell(A) \cap U \neq(0)$. Let $0 \neq u \in \ell(A) \cap U$. Now $A \subseteq$ $r(u) \neq R$ so $A=r(u)$. Clearly $u^{k} x=0$ yields $u^{k-1} x \in r(u) \cap U=A \cap U=(0)$, for any positive integer $k$, and hence $u x=0$. Hence $r(u)=r\left(u^{2}\right)=\cdots$. Now let $I$ be a right ideal of $R$ with $I \cap(u R+r(u))=(0)$. For each $k$ set $T_{k}=u I+u^{2} I+\cdots+u^{k} I$ and observe that $T_{k}$ is an $N$-subgroup. By (3; Thm 6) $R$ has maximum condition on $R$-subgroups so for some $n, T_{n}=T_{n+1}$. Then $u^{n+1} I \subseteq u I+u^{2} I+\cdots+u^{n} I$ and $x \in I$ implies $u^{n+1} x=u t_{1}+u^{2} t_{2}+\cdots u^{n} t_{n}$ where $t_{i} \in I$. We see that $t_{1}+u t_{2}+\cdots+u^{n-1} t_{n} \in$ $r(u)+u R$ and hence $t_{1} \in I \cap(r(u)+u R)=(0)$. Similarly $t_{2} \in\left(r\left(u^{2}\right)+u R\right) \cap I=$ $(r(u)+u R) \cap I=(0)$ and $t_{1}=t_{2}=\cdots=t_{n}=0$. Thus $u^{n+1} I=(0)$ and $I \subseteq r\left(u^{n+1}\right)=r(u)$ from which it follows that $I=(0)$. We then have $r(u)+u R$ module-essential in $N$ and hence $r(u)+u R=R$. Write $1=u t+v, u t \in u R, v \in r(u)$. Then $u=(u t+v) u=u t u+v^{\prime}$ for some $v^{\prime} \in r(u)$ and so $-u t u+u \in u R \cap r(u)=(0)$. Hence $u(1-t u)=0$ and $1-$ $t u \in r(u)$. We see that $R=t u R+r(u)$ and $1=t u+w, w \in r(u)$. Writing $e=t u$ we let $s \in e R \cap r(u)$. Then $s=e y=t u y$ so $u s=u t u y=0$. Using $u=u t u$ we see that $u y=0$ and hence $s=0$ so that $e R \cap r(u)=(0)$. Also if $u a=0$ then $t u a=e a=0$ and $r(u) \subseteq$ $r(e) \neq R$. We see, therefore, that $A=r(e)$. Next we have $e-e^{2}=(e+w) e-e^{2} \in$ $e R \cap r(u)=(0)$ and $e=e^{2} \neq 0$. To prove that $e R$ is minimal we observe that, as an $R$-module, $e R$ is completely reducible so if $e R$ is not minimal we can take non-zero submodules $B, C$ of $e R$ with $e R=B \oplus C$. Then $e=b+c$. If $e x=0$ then $(b+c) x=$ $b x+c x=0$ and thus $b x=0$. Then $r(e) \subseteq r(b) \neq N$ so that $r(e)=r(b)$. If we take $B$ to be minimal then $B=b R$ and $b^{2}=b \neq 0$. Also, $b R \cap r(b)=(0)$ so $b R+r(b)=R$. For $c \in C$ we have $c=b n+z$ where $z \in r(b)$ and $-b n+c \in e R \cap r(b)=e R \cap r(e)=(0)$. Thus $c=b n \in B \cap C=(0)$ and $C=(0)$. Hence we have $e R$ minimal as required.

Lemma 6. If $R$ is semisimple with identity and $A$ is a maximal right annihilator and if $I$ is an $R$-subgroup with $A \neq A+I$ then $A+I=R$.

Proof. From Theorem 8, $A=r(e)$ with $e R$ minimal. Then $R=r(e)+e R=$ $r(e)+I+e R$. Thus if $x \in r(e)+I$ then $x=v+e u(v \in r(e))$ and $e u=-v+x \in$ $e R \cap(A+I)$. If $e R \cap(A+I)=(0)$ then $e u=0$ so $x \in A$ and $A+I \subseteq A$ which is false. Hence $e R \subseteq(A+I)$ so $A+I=R$. 
Theorem 9. Let $R$ be a semisimple near-ring with identity which is the direct sum of ideals each of which is a simple non-ring. Then $R$ has only finitely many maximal right annihilators.

Proof. Let $A$ be a maximal right annihilator and write $R=I_{1} \oplus I_{2} \oplus \ldots \oplus I_{k}$ where each $I_{j}$ is an ideal of $R$ and a simple non-ring. Furthermore, each $I_{j}$ is a direct sum of minimal right ideals of $R$. For one of these minimal right ideals, say $e_{1} R$, we must have $A \cap e_{1} R=(0)$. Without loss of generality we can suppose $e_{1} R \subseteq I_{1}$. If $f R$ is a minimal right ideal contained in $I_{j}$ for some $j>1$ then $A \cap\left(e_{1} R \oplus f R\right) \neq(0)$ so $0 \neq e_{1} u+f v \in A$ and $f v \neq 0$. Also, since $I_{p} I_{q}=(0)$ if $p \neq q$ we see that for each $j>1$, $\left(e_{1} u+f v\right) I_{j}=f v I_{j} \subseteq A$ and since $f v I_{1}=(0) \subseteq A$ we have $f R \subseteq A$. Thus for $j \geqslant 1, I_{j} \subseteq A$. Next we see from Lemma 6 that $A+e_{1} R=R$ so $\left(A \cap I_{1}\right)+e_{1} I_{1}=I_{1}, A \cap I_{1} \cap e_{1} I_{1}=(0)$. From (4; Lemma 8), $A \cap I_{1} \subseteq r_{l_{1}}\left(e_{1}\right)$. It follows that $A \subseteq r\left(e_{1}\right) \neq R$ and thus $A=r\left(e_{1}\right)$.

Now we can prove

Theorem 10. If $N$ has a semisimple near-ring of right quotients then $N$ has finite rank.

Proof. There remains the case where $A_{i} \subset N_{2}$ for an infinite number of $i$. Clearly we may assume that $A_{i} \subset N_{2}$ for all $i$. Furthermore, $T$ is a semisimple near-ring of right quotients of $N_{2}$ and is the direct sum of ideals each of which is a simple non-ring. Apply Lemma 2 to choose $B_{i}$ to be a submodule of $A_{i}$ and consider $A_{1} T$. Since $A_{1} T$ contains $T$-subgroups it contains minimal $T$-subgroups and these have the form $e T$ where $e$ is a non-zero idempotent. Let $e_{1} T$ be a minimal $T$-subgroup contained in $A_{1} T$ and $e_{1}=p_{1} v_{1}^{-1}$ where $p_{1} \in A_{1}$ and $v_{1} \in N_{2}$ is cancellative. Since $e_{1} T+r_{T}\left(e_{1}\right)=T$ we see that $p_{1} N_{2}+r\left(e_{1}\right)$ is $N_{2}$-essential in $N_{2}$ (here and for the remainder of the proof $r(x)$ denotes the right annihilator of $x$ in $N_{2}$ i.e. $r(x)=r_{T}(x) \cap$ $\left.N_{2}\right)$. Furthermore, $A_{2} T \cap r_{T}\left(e_{1}\right) \neq(0)$. Let $e_{2} T$ be a minimal $T$-subgroup of $T$ contained in $A_{2} T \cap r_{T}\left(e_{1}\right)$. Suppose now that we have constructed $e_{1}=p_{1} v_{1}^{-1} \in A_{1} T$, $e_{2}=p_{2} v_{2}^{-1} \in A_{2} T \cap r_{T}\left(e_{1}\right), \ldots, e_{k}=p_{k} v_{k}^{-1} \in A_{k} T \cap r_{T}\left(e_{1}\right) \ldots \cap r_{T}\left(e_{k-1}\right)$ where each $e_{i}$ is a non-zero idempotent and $e_{i} T$ is a minimal $T$-subgroup. Suppose $A_{k+1} \cap r\left(e_{1}\right) \cap \ldots \cap$ $r\left(e_{k}\right)=(0)$. Since $p_{k} N_{2}+r\left(e_{k}\right)$ is $N_{2}$-essential in $N_{2}$ we see that $\left(A_{k} \cap r\left(e_{1}\right) \cap \ldots \cap\right.$ $\left.r\left(e_{k-1}\right)\right)+r\left(e_{k}\right)$ is $N_{2}$-essential in $N_{2}$ and thus $A_{k} \cap r\left(e_{1}\right) \cap \ldots \cap r\left(e_{k-1}\right)$ is $N_{2}$-essential in $A_{k+1} \cap r\left(e_{1}\right) \cap \ldots \cap r\left(e_{k-1}\right)$. Let $0 \neq x \in B_{k+1}$. There is a cancellative element $c \in N_{2} \quad$ with $\quad x c \in\left(p_{1} N_{2}+r\left(e_{1}\right)\right) \cap A_{k+1}=p_{1} N_{2}+A_{k+1} \cap r\left(e_{1}\right)$. Hence for some $s_{1} \in N_{2}$ we have $0 \neq p_{1} s_{1}+x c \in A_{k+1} \cap r\left(e_{1}\right)$. Then $\left(p_{1} s_{1}+x c\right) d_{1}-p_{1} s_{1} d_{1}+p_{1} s_{1} d_{1} \in$ $A_{k+1} \cap r\left(e_{1}\right) \cap\left(p_{2} N_{2}+r\left(e_{2}\right)\right)=p_{2} N_{2}+A_{k+1} \cap r\left(e_{1}\right) \cap r\left(e_{2}\right)$ for some cancellative $d_{1} \in N_{2}$. Let $x_{1}=\left(p_{1} s_{1}+x c\right) d_{1}-p_{1} s_{1} d_{1} \in B_{k+1}$. Then $x_{1} \neq 0$ and for some $s_{2} \in N_{2}$ we have $0=p_{2} s_{2}+p_{1} s_{1}+x_{1} \in A_{k+1} \cap r\left(e_{1}\right) \cap r\left(e_{2}\right) \quad$ so with $a_{1}=p_{1} s_{1} d_{1} \in A_{k}$ and $a_{2}=$ $p_{2} s_{2}+p_{1} s_{1} d_{1} \in A_{2}$ we have, for some cancellative $d_{2} \in N_{2},\left(a_{2}+x_{1}\right) d_{2}-a_{2} d_{2}+a_{2} d_{2} \in$ $A_{k+1} \cap r\left(e_{1}\right) \cap r\left(e_{2}\right) \cap\left(p_{3} N_{2}+r\left(e_{3}\right)\right)$ and we find $x_{2}=\left(a_{2}+x_{1}\right) d_{2}-a_{2} d_{2} \in B_{k+1}, x_{2} \neq 0$ and $a_{3} \in A_{3}$ with $a_{3}+x_{2} \in A_{k+1} \cap r\left(e_{1}\right) \cap r\left(e_{2}\right) \cap r\left(e_{3}\right)$. Continuing in this way we arrive at $a_{k-1} \in A_{k-1}, x_{k-2} \in B_{k+1}$ and $a_{k-1}+x_{k-2} \in A_{k+1} \cap r\left(e_{1}\right) \cap \ldots \cap r\left(e_{k-1}\right)$ and at each stage $x_{i} \neq 0$. For some cancellative $d^{\prime} \in N_{2}$ we now have $\left(a_{k-1}+x_{k-2}\right) d^{\prime}{ }_{k-1} \in$ $A_{k} \cap r\left(e_{1}\right) \cap \ldots \cap r\left(e_{k-1}\right) \subseteq A_{k}$. Hence $\left(a_{k-1}+x_{k-2}\right) d^{\prime}-a_{k-1} d^{\prime} \in A_{k} \cap B_{k+1}=(0)$. But then $x_{k-2}=0$ which is false. Thus if the chain $A_{1} \subset A_{2} \subset \ldots$ does not terminate finitely then 
for each $m, A_{m} \cap r\left(e_{1}\right) \cap \ldots \cap r\left(e_{m-1}\right) \neq(0)$. However, $r_{T}\left(e_{i}\right)$ is a maximal right annihilator in $T, T$ has only finitely many such and their intersection is zero. This contradiction establishes the result.

\section{REFERENCES}

(1) A. W. Goldie, Some aspects of ring theory, Bull. London Math. Soc. (2) 1 (1969), 129-154.

(2) W. M. L. Holcombe, Near-rings of quotients of endomorphism near-rings, Proc. Edinburgh Math. Soc. 19, (1975), 345-352.

(3) A. Oswald, Semisimple near-rings have maximum condition on $N$-subgroups, $J$. London Math. Soc. (2) 11 (1975), 408-412.

(4) A. Oswald, Completely reducible near-rings, Proc. Edinburgh Math. Soc. 20 (1976), 187-197.

(5) G. PILZ, Near-rings (North-Holland, 1977).

(6) L. W. SMALL, Orders in Artinian Rings, J. Algebra 4 (1966), 13-41.

DePaRTMENT OF MATHEMATICS

TeEsside Polytechnic

MidDlesbrough

Cleveland

ENGLAND 